\title{
The Equation Based on the Rotational and Orbital Motion of the Planets
}

\author{
G.A. Korablev \\ Izhevsk State Agricultural Academy, 426069, Russia, Izhevsk, Studencheskaya St.
}

\section{ABSTRACT}

Equations of dependence of rotational and orbital motions of planets are given, their rotation angles are calculated. Wave principles of direct and reverse rotation of planets are established. The established dependencies are demonstrated at different scale levels of structural interactions, in biosystems as well. The accuracy of calculations corresponds to the accuracy of experimental data.

Keywords: orbital and rotational motion of planets, direct and reverse rotation of planets, rotation angles.

\section{INTRODUCTION}

The world around us is in constant motion. Functionally interconnected main types of mechanical motion (translational, rotational and oscillatory) determine the dynamic stability of systems. Vast theoretical and experimental experience in physical and mathematical properties of simple and complex compounds and principles of their self-organization at different scale levels of such conformation has been gained till now.

But the problem of establishing the most general regularities of these processes is topical. "However the science is still far from making it happen in the general form". ${ }^{1}$ Thus, applying the entire set of analytical and qualitative methods, the celestial mechanics provides the solution for many problems on the motion of solids, for example.,3 But some other issues of celestial mechanics require further discussion, for instance, the functional dependence of rotational and orbital motion of planets, as well as the initial principles of forming the direct and reverse motion of planets. Therefore in this paper we attempt to investigate such problematic issues with the help of conception of corpuscular-wave dualism proposed earlier. ${ }^{4}$

\section{INITIAL CRITERIA}

(Based on paper ${ }^{4}$ )

1. In the systems in which the interaction proceeds along the potential gradient (positive work), the resultant potential energy is found based on the principle of adding reciprocals of corresponding energies of subsystems. Similarly, the reduced mass for the relative motion of the isolated systems of two particles is calculated.

2. In the systems in which the interactions proceed against the potential gradient (negative performance) the algebraic addition of their masses, as well as the corresponding energies of subsystems is performed (similar to Hamiltonian).

3. Two principles of adding energy characteristics of structural interactions can be transformed onto the corpuscular-wave dualism processes. Corpuscular processes flow in all interactions along the potential gradient, and wave dualism corresponds to the interactions against the potential gradient.

4. Act of quantum action expressed via Plank's constant is narrowed to the energy equilibriumexchange redistribution between the corpuscular and wave processes.

5. Phase difference of electric and magnetic oscillations in electromagnetic wave is $\pi / 2$. Applying $(2 / \pi)^{2}$ as the proportionality coefficient, we have the equation for Plank's constant with the accuracy close to the accuracy of the initial data:

$\square=\left(\frac{4}{\pi^{2}}+a_{0}\right) P_{e} \frac{\varepsilon}{\mu}$,

where $a_{0}=0.0023293-$ experimental quantum correction to spin $\mathrm{g}_{\mathrm{s}}-$ factor, $\varepsilon$ - electric constant, $\mu-$ magnetic constant. Here: $P_{e}=w r$, where $w$ - energy of a free electron, $r$ - its classic radius.

6. It is assumed that during the rotationaltranslation motion of an electron, the energies are redistributed in the system "particle-wave" that is demonstrated via the angular vector of such motion (winding angle) $-\theta$.

This angular vector of electron motion is quantized by an integer number through the tangent square of this angle: $\operatorname{tg}^{2} \varphi_{2}=2 ; \operatorname{tg}^{2} 60^{\circ}=3 ; \operatorname{tg}^{2} 45^{\circ}=$ 1 , where $\varphi_{2}=54.73^{\circ}-$ a so-called "geodesic angle", which is widely spread in engineering, for example, in spaceship production.

The quantum functions of square tangent $k$ $=1,2,3$ numerically determine the ratios of two triangle legs, whose values characterize energy 
dependencies via axial and circumferential stresses in the system with corpuscular and wave processes.

7. In quantum mechanics the ratio between the particle magnetic moment and its mechanical moment is the magnetomechanic ratio $-g$. At the same time, $g_{s}=2$, if the electron magnetic moment is conditioned only by the spin component and $g=1$, if it is produced by the electron orbital motion. Their ratio $g_{s} / g=2$ that, the same way as $\operatorname{tg}^{2} \varphi_{2}=2$, characterizes the corresponding corpuscular-wave dependencies in this approach.

\section{EQUATION OF DEPENDENCE OF ROTATIONAL AND ORBITAL MOTION OF PLANETS}

The foregoing principles of corpuscularwave mechanism give the possibility to consider from the unified positions many structural and dynamic processes, different in nature and scale. For example, the characteristic of spin-orbital interaction - fine structure constant $\alpha=r / \lambda$, where $r$ - electron classic radius, $\lambda-$ its Compton wavelength.

Formally, but similarly: interaction force of two long conductors with the current proportionally to the ratio $l / 2 \pi r$, where $l$ - length of conductors, $r$-distance between them.

And the number $2 \pi$ widely used in physical regularities, equals the circumference ratio to its radius: $2 \pi=l / R$.

In these examples, as in many other, this approach allows evaluating structural interactions based on the ratios of corpuscular and wave spatialenergy parameters in each action. Obviously, such principles are also demonstrated in Kepler's third law, which can be given as follows ${ }^{3}$ :

$$
G m=4 \pi^{2} \frac{a^{3}}{T^{2}}
$$

where: $G$ - gravitational constant, $m-$ planet mass, here $a$ - distance to barycenter (system mass center), $T$ - planet revolution period. Entering the relative mass $\quad m_{0}=m / m_{3}$ (where $m_{3}-$ Earth mass), we can have:

$$
\begin{aligned}
& \frac{G m_{2}}{4 \pi^{2}}=\frac{a^{3}}{T^{2} m_{0}} \text {, therefore } \frac{a^{3}}{T^{2} m_{0}}=\text { const } \\
& \text { Or: } \quad \frac{a}{\left(T^{2}\right)^{\frac{1}{3} m_{0}} \frac{1}{3}}=\text { const }
\end{aligned}
$$

Since the masses of planets are rather small in comparison with their distance to the sun, then at the first approximation they can be considered as mathematical points and the equation of mathematical pendulum period can be applied to them:

$T^{2}=4 \pi^{2} \frac{\mathbb{I}}{g}$,

where $g$ - free fall acceleration. The average radius of the planet orbital motion $-R$ can be taken as the pendulum length $-l$. Then, similarly to the foregoing dependencies, we can introduce the planet radius $r$ into the numerator of formula (2) and then we have:

$$
\frac{\gamma}{\left(\frac{4 \pi^{2}}{g} R m_{0}\right)^{\frac{1}{3}}}=\gamma
$$

This expression in the units $m / \sec ^{2 / 3}$ satisfies the principle of corpuscular-wave mechanism for space macrostructures. But in Kepler's third law only the orbital motion was considered, but in this case - two motions, each of which has its own wave part. Therefore, the interference of coherent waves occurs.

Similar to the foregoing examples, the coherence can be considered as the ratio of the difference of the travel path to the length of the coherent wave $(\Delta / \lambda)$. The interference principle is most easily performed for liquid-gaseous planets (planets in Jupiter systemпланеты системы Юпитера) as shown in Table 1:

$$
\beta_{+}=2 n \frac{Y}{2}
$$

- boosting of oscillations during the direct rotation of planets.

$$
\beta_{-}=(2 n+1) \frac{Y}{2}
$$

- damping of oscillations during the reverse rotation of planets.

Here $n$-integer number.

The intensity of wave propagation depends on the medium density and its distribution in the planet volume.

The value characterizing the planet density increase towards its center is called "dimensionless moment of inertia" $\left(I^{*}\right)$. The ratio of average values of $\mathrm{I}^{*}$ for solid and liquid-gaseous planets based on different data ${ }^{3,5,6}$ is within $1.4-1.45$ as demonstrated in Table 2.

Table 1- Characteristics of rotational and orbital motion of planets 

ISSN : 2248-9622, Vol. 7, Issue 3, ( Part -2) March 2017, pp.69-72

\begin{tabular}{|c|c|c|c|c|c|c|c|c|c|c|c|}
\hline Planet & $\mathbf{r} \cdot 10^{6}, \mathrm{~m}$ & $\mathbf{R} \cdot \mathbf{1 0}^{9}, \mathrm{~m}$ & $\gamma, \frac{m}{\sec ^{2 / 3}}$ & $\begin{array}{l}\text { Direction } \\
\text { of rotation }\end{array}$ & $\begin{array}{l}\text { Calculation } \\
\text { formula for } \beta\end{array}$ & $\mathbf{n}$ & $\beta, \frac{m}{\sec ^{2 / 3}}$ & $\boldsymbol{\delta}=\frac{\boldsymbol{\beta}}{\boldsymbol{\beta}_{\mathbf{o}}}$ & $\theta_{1}^{\circ}$ & $\mathbf{\theta}_{2}^{\circ}$ & $\boldsymbol{\theta}^{\circ} 3,5,6$ \\
\hline Mercury & 2.4397 & 57.9 & 1039.7 & + & $n^{1 / 2} \frac{\gamma}{2}$ & 2 & 735.2 & 0.9735 & & 86.6 & 87.0 \\
\hline Venus & 6.0515 & 108.2 & 855.17 & - & $(2 n+1)^{1 / 2} \frac{\gamma}{2}$ & 1 & 740.6 & 0.9810 & & 87.2 & 87.0 \\
\hline Earth & 6.3780 & 149.6 & 755.2 & + & $2 n^{1 / 2} \frac{\gamma}{2}$ & 1 & 755.2 & 1 & 66.56 & & 66.556 \\
\hline Mars & 3.3970 & 227.9 & 734.7 & + & $2 n^{1 / 2} \frac{\gamma}{2}$ & 1 & 734.7 & 0.9728 & 64.75 & & 64.8 \\
\hline Jupiter & 71.492 & 778.6 & 715.8 & + & $2 n \frac{\gamma}{2}$ & 1 & 715.8 & $0.9478^{1 / 2}$ & & 86.6 & 86.9 \\
\hline Saturn & 60.268 & 1433.7 & 735.9 & + & $2 n \frac{\gamma}{2}$ & 1 & 735.9 & 0.9744 & 64.85 & & 64.3 \\
\hline Uranus & 25.596 & 2870.4 & 463.6 & - & $(2 n+1) \frac{\gamma}{2}$ & 1 & 696.8 & 0.9227 & & 82.07 & 82.0 \\
\hline Neptune & 24.764 & 4491.1 & 365.49 & + & $2 n \frac{\gamma}{2}$ & 2 & 730.98 & $0.96793^{2}$ & 62.5 & & 61.68 \\
\hline Pluto & 1.1510 & 5868.9 & 295.55 & - & $(2 n+1) \frac{\gamma}{2}$ & 2 & 738.75 & 0.9782 & & & \\
\hline & & & & & & & $\langle\beta\rangle=732.5$ & & & & \\
\hline
\end{tabular}

Table 2 - Ratio of dimensionless moments of inertia of solid and liquid-gaseous planets ${ }^{3,5}$

\begin{tabular}{|l|l|l|l|l|l|}
\hline Planet & Mercury & Venus & Earth & Mars & $\left\langle\mathbf{I}_{\mathbf{S}}^{*}\right.$ \\
\hline $\mathbf{I}_{\mathbf{S}}^{*}$ & 0.324 & 0.333 & 0.33076 & 0.377 & $\mathbf{0 . 3 4 1}^{*}$ \\
\hline Planet & Jupiter & Saturn & Uranus & Neptune & ${\left\langle\mathbf{I}_{\mathbf{L G}}^{*}\right.}^{\mathbf{*}}$ \\
\hline $\mathbf{I}_{\mathbf{L G}}^{*}$ & 0.20 & 0.22 & 0.23 & 0.29 & $\mathbf{0 . 2 3 5}$ \\
\hline
\end{tabular}

The average value $\mathrm{I}_{\mathrm{S}} / \mathrm{I}_{\mathrm{LG}}^{*}=1.451$

Such property for solid planets is taken into account in Table 1 and equations $(4,5)$ by introducing the values $n^{1 / 2}$ and $(2 n+1)^{1 / 2}$. Such approach also refers to Mercury as it is the nearest to the sun, to its liquid-gaseous structure.

In general, the application of corpuscularwave mechanism to space macrosystems explains the specifics of formation of direct or reverse rotation of the planets.

\section{ROTATION ANGLES OF PLANETS}

In physical sense, the parameter $\beta$ characterizes the motion difference of interfering waves, and $\gamma$-wavelength. The average value of $\beta$ $=732.5 \mathrm{~m} / \mathrm{sec}^{2 / 3}$ with the deviation of most of the planets under $2 \%$ (apart from Uranus).

The equation $\operatorname{tg}^{2} \theta=k$ was used to evaluate quantum transitions in atoms in. ${ }^{4}$ The squares and cubes of initial parameters are applied in Kepler's equation and other regularities of space macrosystems. In this approach, as the calculations demonstrated, the semi-empirical equation is performed:

$$
\beta=\left(\operatorname{tg}^{2} \theta\right)^{2}
$$

where $\theta$ - rotation angle of planets.

For Earth $\beta=755.2$ and based on equation (6) $\theta_{0}=66.455^{\circ}$. For more accurate calculation, taking into account some analogy of macro- and microprocesses, we use, as before, the experimental quantum correction in the form of $a_{0}=1.0023293$ following the equation:

$$
\theta_{1}=a_{0} \theta_{0}
$$

The calculation by equations for Earth (6, 7) gives the value $\theta_{1}=66.560^{\circ}$ with the deviation from the experimental value by $0.007 \%$. The sun has the same value of the rotation angle.

As applicable to the rest of the planets, the value $\delta=\beta / \beta_{0}$, (where $\beta_{0}-\beta$ value for Earth) is introduced in equation (7) based on the following equations:

$$
\begin{array}{cl}
\theta_{1}=a_{0} \delta \theta_{0} & \text { or } \theta_{1}=a_{0} \frac{\beta}{\beta_{0}} \theta_{0} \\
\theta_{2}=\frac{4}{a} a_{0} \delta \theta_{0} & \text { or } \theta_{2}=\frac{4}{a} a_{0} \frac{\beta}{\beta_{0}} \theta_{0}
\end{array}
$$

The formulas ( 8 and $8 \mathrm{a})$ are performed for the planets, whose rotation angle is less than Earth's one. For the other planets the formulas (9 and 9a) are performed. Those are the planets, which are in the beginning of the planet subsystem by the value of the dimensionless moment of inertia (Mercury and Jupiter), as well as the planets with the reverse rotation (Venus and Uranus). The calculation results are given in Table 1 .

The coefficient $4 / 3$ has been applied before for the comparative evaluation of quantum transitions with different complexity types ${ }^{4}$. In this research the average ratio of the angles by the experimental data ${ }^{3,5,6}$ given in Table 3 also has the value $1.336 \approx 4 / 3$.

Table 3 - Ratio of rotation angles of planets by $\mathrm{y}^{3,5,6}$

\begin{tabular}{|l|l|l|l|l|l|}
\hline Planet & Mercury & Venus & Earth & Mars & $\left\langle\square_{\mathbf{2}}\right\rangle$ \\
\hline$\square_{\mathbf{2}}$ & 87.00 & 87.00 & 86.90 & 82.00 & $\mathbf{8 5 . 7 3}$ \\
\hline Planet & Jupiter & Saturn & Uranus & Neptune & $\left\langle\square_{\mathbf{1}^{\rangle}}\right.$ \\
\hline$\square_{\mathbf{1}}$ & 66.556 & 64.80 & 64.30 & 61.00 & $\mathbf{6 4 . 1 6}$ \\
\hline
\end{tabular}

The average value $\Theta_{2} / \Theta_{1}=1.336 \approx 4 / 3$.

For the value $\delta$ the influence of the medium density distribution is taken into account via the transition factor from one distribution level to another. Since for Jupiter in Table 2 the value $I^{*}$ is less in comparison with Earth's $I^{*}$ in 1.45 times, therefore, in the calculations $\delta=0.9478^{1 / 2}$. On the contrary with Jupiter, for Neptune $I^{*}$ increases in 
1.45 times, therefore, in the calculations $\delta=$ $0.96793^{2}$.

All the calculation results are in good accord with the experimental data.

\section{CONCLUSIONS}

1. Semi-empirical equations of the dependence of rotational and orbital motion of planets are obtained.

2. Many structural-dynamic processes in macroand microsystems, including the specifics of formation of direct or reverse rotation of the planets are explained based on the previously proposed method to evaluate the corpuscularwave mechanism.

3. The given calculations of rotation angles of the planets are within the accuracy of the experimental data.

\section{REFERENCES}

[1]. Erden-Gruz, T. Basics of matter composition. Moscow, 1976, 438.

[2]. Duboshin, G.N. Celestial mechanics. Nauka, Moscow, 1978, 456.

[3]. Panteleev, V.L. Physics of Earth and planets. Lectures. MSU. Moscow, http://www.astronet.ru/db/msg/1169697/n ode17.html

[4]. Korablev, G.A. Spatial-energy parameter and its application in research. LAP LAMBERT Academic Publishing. Germany, 2016, 1-65.

[5]. Encyclopedia in physics. Moscow, 1988, 1, 704; 1990, 2, 704; 1992, 3, 672.

[6]. Allen, K.U. Astrophysical magnitudes. Mir, Moscow, 1977, 446.

\section{Information about the author}

Grigory Andreevich Korablev - Professor, Doctor of Science in Chemistry, Head of Physics Department at Izhevsk State Agricultural Academy. 